\title{
Probing Spin Dependent Quark and Gluon Distributions Through Azimuthal and Polarization Asymmetries
}

\author{
Gary R. Goldstein ${ }^{1, a}$ and Simonetta Liuti ${ }^{2,3, b}$ \\ ${ }^{1}$ Department of Physics and Astronomy, Tufts University, Medford, MA 02155 USA. \\ ${ }^{2}$ Department of Physics, University of Virginia, Charlottesville, VA 22904, USA. \\ ${ }^{3}$ Laboratori Nazionali di Frascati, INFN, Frascati, Italy
}

\begin{abstract}
Spin and transverse momentum dependent parton distributions - Generalized Parton Distributions, Transverse Momentum Distributions and related distributions - are at the interface between the QCD structure of the hadrons and observable quantities. The distributions are contained as linear superpositions within helicity amplitudes that factorize into universal forms at leading or next to leading order. These amplitudes are probed in high energy leptoproduction processes through angular dependent cross sections and polarization asymmetries. The phenomenological extraction of the amplitudes and the distributions is a challenging task. We will present some models for the distributions in leptoproduction processes, as well as the observables that connect with the quark-nucleon helicity amplitudes for different Deeply Virtual production possibilities.
\end{abstract}

\section{Introduction}

The spin of the hadrons depends on the distributions of spin and orbital angular momenta (OAM) of the fundamental constituents, quarks and gluons. The quark and gluon field correlations in the nucleon are indirectly measurable through electroproduction processes. The angular momenta associated with the quark and gluon fields within QCD, are encoded in the transverse momentum distributions (TMDs) and the Generalized Parton Distributions (GPDs). These can provide a 3-dimensional picture of the angular momentum structure.

Of special interest among pdf's are the nucleon's transversity structure functions, $h_{1}(x)$ - the probability of finding a definite transversity quark inside a transversely polarized nucleon. They are chiral odd, and can be observed in Semi Inclusive Deep Inelastic Scattering (SIDIS), where they are convoluted with fragmentation functions, or in the Drell-Yan process in conjunction with another chiral-odd partner. They also contribute to exclusive electroproduction processes, particularly Deeply Virtual Meson Production (DVMP), through chiral odd GPDs. The transversity GPD $H_{T}^{q}(x, \xi, t)$ has the limiting form $H_{T}^{q}(x, 0,0)=h_{1}^{q}(x)$. Hence, from the DVMP processes, transversity can be determined. This will be a main focus of this presentation.

In advance of the development of the formalism and the brief presentation of our model, we show some important results - the transversity $h_{1}(x)$ in Fig 1(a) and the predicted asymmetry $A_{U T}^{\sin \left(\phi-\phi_{s}\right)}\left(x_{B j}, t, Q^{2}\right)$, that is particularly sensitive to the transversity, in Fig. 1(b).

\footnotetext{
a e-mail: gary.goldstein@tufts.edu

be-mail: sl4y@virginia.edu
} 

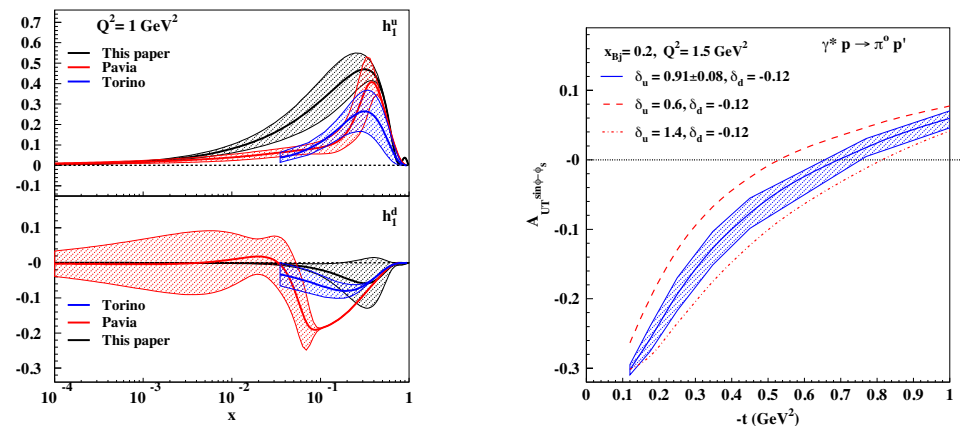

Figure 1. Left (a): transversity, $h_{1}^{q}$ plotted vs. $x$ at $Q^{2}=1 \mathrm{GeV}^{2}$, for the $u$ quarks (upper panel) and for the $d$ quark (lower panel). Besides our analysis, the recent extractions from the Pavia group [1] obtained from dihadron production in a collinear framework, and from the Torino group [2] obtained combining data on polarized SIDIS single hadron production [3, 4], and dihadron production from $e^{+} e^{-}$annihilation [5]. Right (b): asymmetry $A_{U T}^{\sin \left(\phi-\phi_{S}\right)}$, plotted vs. $-t$, at $x_{B j}=0.2, Q^{2}=1.5 \mathrm{GeV}^{2}$ for the $\gamma^{*} p \rightarrow \pi^{0} p^{\prime}$ reaction. The graph shows the sensitivity of the asymmetry to variations of the tensor charge, or the precision that is needed in measurements of this quantity in order to reduce the size of the errors. Adapted from Ref. [6].

\section{Formalism}

DVCS and DVMP can be described within QCD factorization, through the convolution of specific GPDs and hard scattering amplitudes. There are four chiral-even GPDs, $H, E, \widetilde{H}, \widetilde{E}$ [9] and four additional chiral-odd GPDs, known to exist by considering twist-two quark operators that flip quark helicity by one unit, $H_{T}, E_{T}, \widetilde{H}_{T}, \widetilde{E}_{T}[10,11]$. All GPDs depend on two additional kinematical invariants besides the parton's Light Cone (LC) momentum fraction, $x$, and the DVCS process' four-momentum transfer, $Q^{2}$, namely $t=\Delta^{2}$ where $\Delta=P-P^{\prime}$ is the momentum transfer between the initial and final protons, and $\xi$, or the fraction of LC momentum transfer, $\xi=\Delta^{+} /\left(P^{+}+P^{\prime+}\right)$. The observables containing the various GPDs are the Compton Form Factors (CFFs) - convolutions over $x$ of GPDs with the struck quark propagator. The quark GPDs are defined (at leading twist) as the matrix elements of the following projection of the unintegrated quark-quark proton correlator (see Ref.[11] for a detailed overview),

$$
W_{\Lambda^{\prime}, \Lambda}^{\Gamma}(x, \xi, t)=\left.\frac{1}{2} \int \frac{d z^{-}}{2 \pi} e^{i x \bar{P}^{+} z^{-}}\left\langle P^{\prime}, \Lambda^{\prime}\left|\bar{\psi}\left(-\frac{z}{2}\right) \Gamma \psi\left(\frac{z}{2}\right)\right| P, \Lambda\right\rangle\right|_{z^{+}=0, \mathbf{z}_{T}=0},
$$

where $\Gamma=\gamma^{+}, \gamma^{+} \gamma_{5}, i \sigma^{i+} \gamma_{5}(i=1,2)$, and the target's spins are $\Lambda, \Lambda^{\prime}$.

For the chiral-odd case, $\Gamma=i \sigma^{i+} \gamma_{5}, W_{\Lambda^{\prime}, \Lambda}^{\Gamma}$ was parametrized as [11],

$$
\begin{aligned}
W_{\Lambda^{\prime}, \Lambda}^{\left[i \sigma^{i+} \gamma_{5}\right]}(x, \xi, t) & =\frac{1}{2 \bar{P}^{+}} \bar{U}\left(P^{\prime}, \Lambda^{\prime}\right)\left(i \sigma^{+i} H_{T}(x, \xi, t)+\frac{\gamma^{+} \Delta^{i}-\Delta^{+} \gamma^{i}}{2 M} E_{T}(x, \xi, t)\right. \\
& \left.+\frac{P^{+} \Delta^{i}-\Delta^{+} P^{i}}{M^{2}} \widetilde{H}_{T}(x, \xi, t)+\frac{\gamma^{+} P^{i}-P^{+} \gamma^{i}}{2 M} \widetilde{E}_{T}(x, \xi, t)\right) U(P, \Lambda)
\end{aligned}
$$

The spin structures of GPDs that are directly related to spin dependent observables are most effectively expressed in term of helicity dependent amplitudes, developed extensively for the covariant description of two body scattering processes (see also Ref.[11]). 

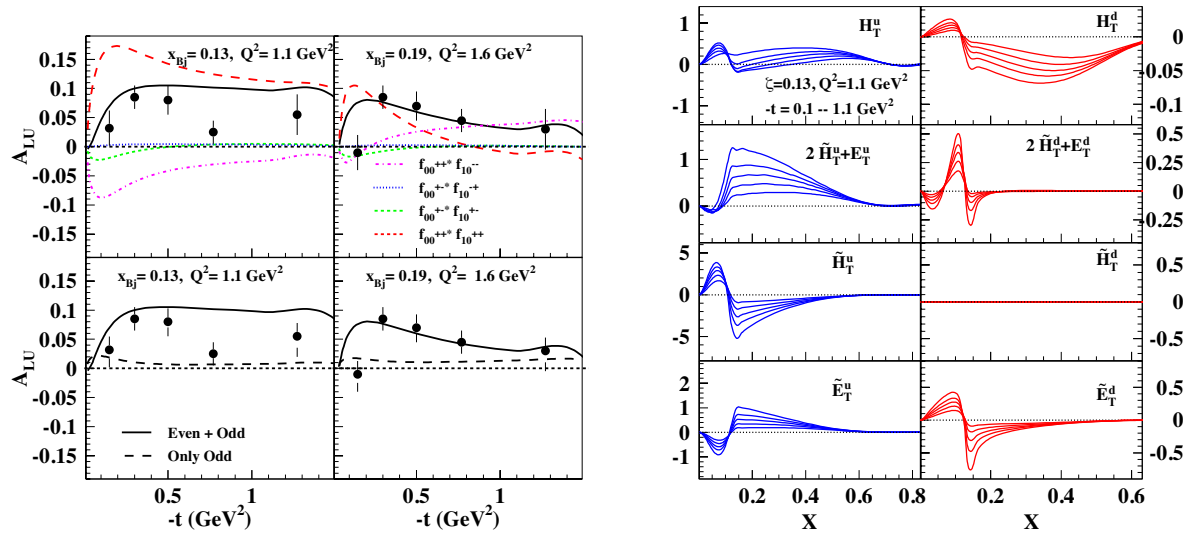

Figure 2. (Color online) Left (a): Beam spin asymmetry, $A_{L U}$, plotted vs. $-t$ for two different kinematics. Experimental data from Ref.[7]. Adapted from Ref. [8]. Right (b): The chiral-odd GPDs evaluated using the model described in the text plotted vs. $\mathrm{X}$ at $x_{B j}=\zeta=0.13, Q^{2}=2 \mathrm{GeV}^{2}$. The range in $-t$ is: $0.1 \leq-t \leq$ $1.1 \mathrm{GeV}^{2}$. Curves with the largest absolute values correspond to the lowest $t$. Adapted from Ref. [8]

We see that there are 8 quark GPDs per flavor (at leading twist). Correspondingly, the quarknucleon amplitudes have the form $\mathrm{N} \rightarrow \mathrm{q}:: \mathrm{q}^{\prime} \rightarrow \mathrm{N}^{\prime}$ with 8 independent helicity amps. There are two questions to address: How to model the 8 GPDs? How to measure them? DVCS accesses Chiral Even GPDs through various cross sections and asymmetries. The GPDs, or their corresponding Compton Form Factors, enter linearly via Bethe-Heitler $\otimes$ DVCS interference. DV $\pi^{0} \mathrm{~S}$ accesses 2 Chiral Even +4 Chiral Odd GPDs that enter bilinearly via $d \sigma / d \Omega \&$ polarization asymmetries. The result of experimental observations that $d \sigma_{T}>d \sigma_{L}$ is that the chiral odd GPDs dominate.

In Ref.[12], after showing how $\mathrm{DV} \pi^{0} \mathrm{P}$ can be described in terms of chiral-odd GPDs, we estimated all of their contributions to the various observables with particular attention to the ones which were sensitive to the values of the tensor charge. Subsequently, an extension of the Flexible Reggeized Diquark model in the chiral-even sector in Refs. $[13,14]$ to the chiral odd sector was accomplished through the use of Parity and Charge Conjugation symmetry relations obeyed by the various helicity structures in the model.The chiral even GPDs integrate to the nucleon form factors, which constrains the GPD t-dependence,

$$
\int_{0}^{1} H^{q}(X, \zeta, t)=F_{1}^{q}(t), \int_{0}^{1} E^{q}(X, \zeta, t)=F_{2}^{q}(t), \int_{0}^{1} \widetilde{H}^{q}(X, \zeta, t)=G_{A}^{q}(t), \int_{0}^{1} \widetilde{E}^{q}(X, \zeta, t)=G_{P}^{q}(t) .
$$

where $F_{1}^{q}(t)$ and $F_{2}^{q}(t)$ are the Dirac and Pauli form factors for the quark $q$ components in the nucleon. $G_{A}^{q}(t)$ and $G_{P}^{q}(t)$ are the axial and pseudoscalar form factors. Furthermore, $H(x, 0,0)=h_{1}(x)$ and $\widetilde{H}(x, 0,0)=g_{1}(x)$.

\section{Helicity, model parameters}

The connection of the correlator, Eq.(2), with the helicity amplitudes proceeds by introducing [8, 12],

$$
f_{\Lambda_{\gamma} 0}^{\Lambda \Lambda^{\prime}}(\zeta, t)=\sum_{\lambda, \lambda^{\prime}} g_{\Lambda_{\gamma} 0}^{\lambda \lambda^{\prime}}\left(X, \zeta, t, Q^{2}\right) \otimes A_{\Lambda^{\prime} \lambda^{\prime}, \Lambda \lambda}(X, \zeta, t)
$$


where the helicities of the virtual photon and the initial proton are, $\Lambda_{\gamma}, \Lambda$, and the helicities of the produced pion and final proton are 0 , and $\Lambda^{\prime}$, respectively. This describes a factorization into a "hard part", $g_{\Lambda_{\gamma} 0}^{\lambda \lambda^{\prime}}$ for the partonic subprocess $\gamma^{*}+q \rightarrow \pi^{0}+q$, and a "soft part" given by the quarkproton helicity amplitudes, $A_{\Lambda^{\prime}, \lambda^{\prime} ; \Lambda, \lambda}$ that contain the GPDs. The amplitudes $A_{\Lambda^{\prime} \lambda^{\prime}, \Lambda \lambda}$ implicitly contain an integration over the unobserved quark's transverse momentum, $k_{T}$, and are functions of $x_{B j}=$ $Q^{2} / 2 M v \approx \zeta, t$ and $Q^{2}$. The convolution integral in Eq.(4) is given by $\otimes \rightarrow \int_{-\zeta+1}^{1} d X$.

The expressions for the chiral-odd helicity amplitudes in terms of GPDs [11] are of the form

$$
A_{++,--}=\sqrt{1-\xi^{2}}\left[H_{T}+\frac{t_{0}-t}{4 M^{2}} \widetilde{H}_{T}-\frac{\xi^{2}}{1-\xi^{2}} E_{T}+\frac{\xi}{1-\xi^{2}} \widetilde{E}_{T}\right], \ldots
$$

where we use the symmetric notation for the kinematic variables. Analogous forms have been written for the chiral even and odd sectors [11].

For a transverse photon, inserting the expressions for $g_{10}^{+-}$and the $A$ 's into Eqs.(4) we obtain a set of helicity amplitudes that enter the observables. They include

$$
f_{10}^{+-}=\frac{g_{\pi}^{V, o d d}(Q)+g_{\pi}^{A, o d d}(Q)}{2} \sqrt{1-\xi^{2}}\left[\mathcal{H}_{T}+\frac{t_{0}-t}{4 M^{2}} \widetilde{\mathcal{H}}_{T}-\frac{\xi^{2}}{1-\xi^{2}} \mathcal{E}_{T}+\frac{\xi}{1-\xi^{2}} \widetilde{\mathcal{E}}_{T}\right]
$$

and analogous terms $([8,12])$, where $\mathcal{H}_{T}$, etc., are the Compton form factors. In the $t$-channel picture, which has its roots in a Regge analysis of this process [15], one separates the $J^{P C}=1^{--}$and $J^{P C}=$ $1^{+-}$contributions to the amplitudes for transverse and longitudinal virtual photons, respectively, thus generating two different types of $Q^{2}$ dependence at the $\pi^{0}$ vertex [16].

For a longitudinal photon one has the convolution of $g_{00}^{+-}$with the $A$ helicity amplitudes. There are contributions for the longitudinal photon from the chiral even $\mathcal{H}, \mathcal{E}$ as well (see Ref. [8]). Following from the chiral-even case, in $\pi^{0}$ electroproduction one obtains longitudinal photon amplitudes [17] that are significant. (see Ref. $[8,13]$ ).

Our model for evaluating the chiral-odd GPDs extends our reggeized diquark model, which was already configured for chiral-even GPDs, to the chiral-odd sector. The parameters were initially fixed by a fit applied recursively first to PDFs, and to the nucleon form factors. The model was shown to reproduce data on different observables in DVCS (charge [18], longitudinal [19] and transverse [18] single spin asymmetries). A comparison with data from more recent analysis has also been shown in Ref.[20]. Recently [14], we presented a new fit that uses the form factor flavor separated data from Ref.[21].

The basic structures in our model are the quark-proton scattering amplitudes at leading order with proton-quark-diquark vertices. The quark parton helicity amplitudes describe a two body process, $q^{\prime}\left(k^{\prime}\right) P \rightarrow q(k) P^{\prime}$, where $q(k)$ corresponds to the "struck quark". The intermediate diquark system, $X$, can have $J^{P}=0^{+}$(scalar), or $J^{P}=1^{+}$(axial vector). We start from the region $X \geq \zeta$. At fixed $M_{X}$, the amplitudes read for the Scalar diquark (see $[12,13]$ for the full set of relations):

$$
A_{\Lambda^{\prime} \lambda^{\prime}, \Lambda \lambda}^{(0)}=\int d^{2} k_{\perp} \phi_{\Lambda^{\prime} \lambda^{\prime}}^{*}\left(k^{\prime}, P^{\prime}\right) \phi_{\Lambda \lambda}(k, P) .
$$

Next we consider reggeization that is, we extend the diquark model formalism to low $X$ by allowing the spectator system's mass to vary up to very large values. This is accomplished by convoluting the GPD structures obtained in Eqs.(7) with a "spectral function", $\rho\left(M_{X}^{2}\right)$, where $M_{X}^{2}$ is the spectator's mass,

$$
F_{T}^{q}(X, \zeta, t)=\mathcal{N}_{q} \int_{0}^{\infty} d M_{X}^{2} \rho\left(M_{X}^{2}\right) F_{T}^{\left(m_{q}, M_{\Lambda}^{q}\right)}\left(X, \zeta, t ; M_{X}\right)
$$



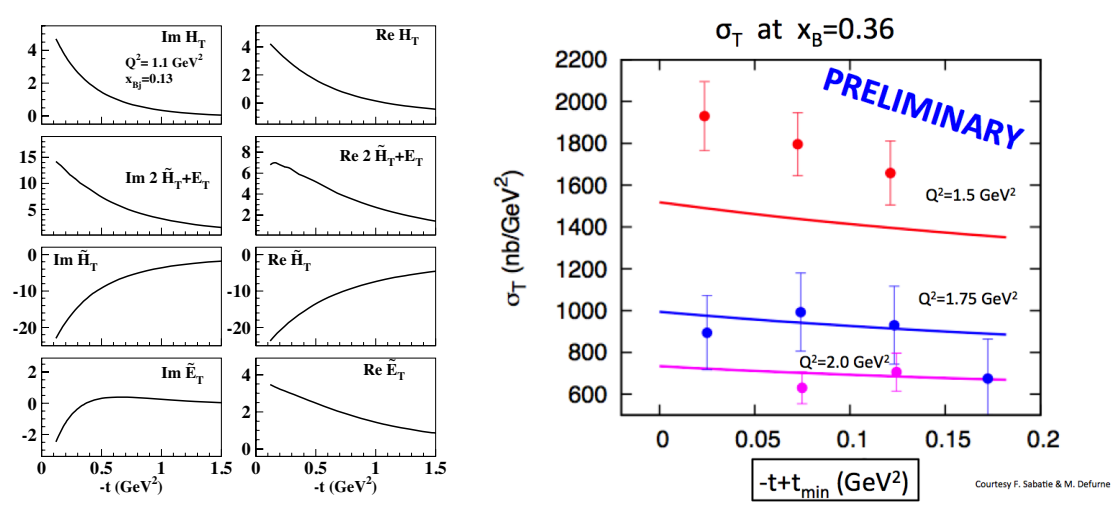

Figure 3. Left (a): Chiral-odd CFFs entering the process $\gamma^{*} p \rightarrow \pi^{0} p^{\prime}$. The various CFFs are plotted vs. $-t$ for the kinematic bin $x_{B j}=0.13, Q^{2}=1.1 \mathrm{GeV}^{2}$. Adapted from Ref. [8]. Right (b): Transverse differential cross section vs. $\left(-t+t_{\min }\right)$ for $Q^{2}=1.5,1.75,2.0 \mathrm{GeV}^{2}$ at $x_{B j}=0.36$. Preliminary data from Hall A, courtesy $\mathrm{F}$. Sabatie \& M. Defurne [23].

The spectral function was constructed in Refs.[13, 14] so that it approximately behaves as $\left(M_{X}^{2}\right)^{\alpha}$ for $M_{X}^{2} \rightarrow \infty$ and $\delta\left(M_{X}^{2}-\bar{M}_{X}^{2}\right)$ for $M_{X}^{2}$ at a few $G e V^{2}$, where $0<\alpha<1$, and $\bar{M}_{X}$ is in the GeV range. We parametrized this in a practical form as,

$$
F_{T}^{q}(X, \zeta, t) \approx \mathcal{N}_{q} X^{-\alpha_{q}+\alpha_{q}^{\prime}(X) t} F_{T}^{\left(m_{q}, M_{\Lambda}^{q}\right)}\left(X, \zeta, t ; \bar{M}_{X}\right)=R_{p_{q}}^{\alpha_{q}, \alpha_{q}^{\prime}}(X, \zeta, t) G_{M_{X}, m}^{M_{\Lambda}}(X, \zeta, t)
$$

where $\alpha_{q}^{\prime}(X)=\alpha_{q}^{\prime}(1-X)^{p_{q}}$. The functions $G_{M_{X}, m_{q}}^{M_{\Lambda}^{q}}$ and $R_{p_{q}}^{\alpha_{q}, \alpha_{q}^{\prime}}$ are the quark-diquark and Regge contributions, respectively.

\section{Observables \& Data}

The fitting procedure of GPDs is quite complicated owing to its many different steps: 1) the construction of chiral-odd helicity amplitudes; 2) the connection of these amplitudes to the chiral-even ones using Parity relations within spectator models; 3) the fixing of chiral-even parameters at an initial scale, $Q_{o}^{2}$, using the nucleon form factors and PQCD evolution to match DIS data; 4) the determination of chiral-odd GPDs; 5) the construction of the corresponding Compton form factors, and pseudoscalar meson electroproduction observables. In the following figures we show the GPDs at various kinematic ranges and some of the CFFs. A more detailed description of the other transversity functions including the first moment of $h_{1}^{\perp} \equiv 2 \widetilde{H}_{T}^{q}+E_{T}^{q}$ is given in [8].

In Fig.2 (b) we show the $t$-dependent chiral odd GPDs that enter the helicity amplitudes evaluated in Eqs. 5 in a kinematical bin $\left(x_{B j}=0.13, Q^{2}=1.1 \mathrm{GeV}^{2}\right)$ consistent with the Jefferson Lab kinematical coverage.

In Fig.3(a) we show the proton CFFs, Eq.(6), which enter the $\gamma^{*} p \rightarrow \pi^{0} p^{\prime}$ reaction. The various GPDs enter the helicity amplitudes and those, in turn, determine all the cross section terms for $\pi^{0}$ electroproduction. The transverse and longitudinal cross sections have been separated experimentally at small $\mathrm{t}$ [23]. Some preliminary data compare favorably with our predictions in Fig 3(b).

(1) All GPD contributions should be considered separately. In particular, $H_{T}, \widetilde{H}_{T}$, and $E_{T}$ are dominating; $\widetilde{E}_{T}$ is non zero in our model but small. Although the combination $2 \widetilde{H}_{T}+E_{T}$ might be considered 

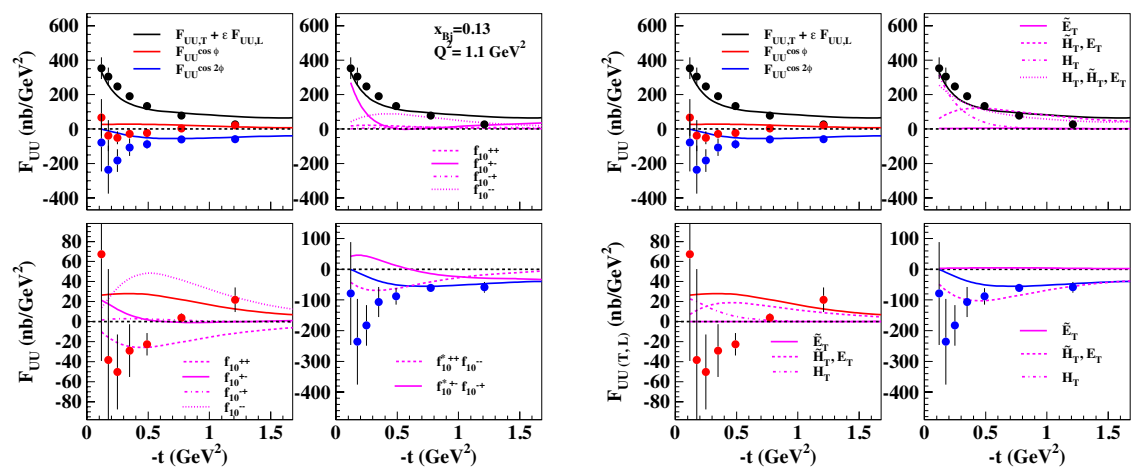

Figure 4. (Color online) LEFT: Unpolarized cross section components, $F_{U U, T}+\epsilon F_{U U, L}, F_{U U}^{\cos 2 \phi}$, and $F_{U U}^{\cos \phi}$ in the kinematical bin, $x_{B j}=0.13, Q^{2}=1.2 \mathrm{GeV}^{2}$. The upper left panel shows all components along with the data from Ref.[22]. The other panels show the contributions from the various helicity amplitudes. The right upper panel similarly shows $F_{U U, T}+\epsilon F_{U U, L}$. See Ref. [8], from which these are adapted, for details.

more fundamental in that its spin structure corresponds to the Boer-Mulders function [11]. $\widetilde{H}_{T}$, and $E_{T}$ appear separately, and are multiplied by different factors in the amplitudes. $2 \widetilde{H}_{T}+E_{T}$ should just be viewed as a forward limit.

(2) The behavior of $f_{10}^{++}$and $f_{10}^{--}$is determined by $\widetilde{H}_{T}$, and $E_{T}$. As a consequence of point $(1), f_{10}^{--}$is sensibly different from $f_{10}^{++}$. In particular, because of the different multiplicative factors, $f_{10}^{--}<f_{10}^{++}$.

(3) $f_{10}^{+-}$is determined by $H_{T}$ at small $|t|$, and by $E_{T}$ at large $|t|$.

(4) $f_{10}^{-+}$is determined by $\widetilde{H}_{T}$ only, but it is small due to the $|t|$ factor suppression.

(5) The longitudinal photon contributions, $f_{00}^{+-}$, and $f_{00}^{++}$are suppressed in the chiral-odd case.

In Fig. 4 we show unpolarized cross section components, $F_{U U, T}+\epsilon F_{U U, L}, F_{U U}^{\cos 2 \phi}$, and $F_{U U}^{\cos \phi}$ as functions of $t$, for the kinematics $x_{B j}=0.13, Q^{2}=1.2 \mathrm{GeV}^{2}$. The caption indicates the different contributions.

The unpolarized $\sin \phi$ modulation, $F_{L U}^{\sin \phi}$ describes the beam asymmetry, $A_{L U}$,

$$
A_{L U}=\sqrt{\epsilon(1-\epsilon)} \frac{F_{L U}^{\sin \phi}}{F_{U U, T}+\epsilon F_{U U, L}}
$$

$A_{L U}$ is shown in Fig.2(a) for two of the Jefferson Lab Hall B kinematical bins along with the different amplitudes contributions, in this case the products: $\left(f_{10}^{++*} f_{00}^{++}\right),\left(f_{10}^{--*} f_{00}^{++}\right),\left(f_{10}^{-+*} f_{00}^{+-}\right)$and $\left(f_{10}^{+-*} f_{00}^{+-}\right)$. Notice that the longitudinally polarized amplitudes receive contributions from both the chiral-even and chiral-odd GPDs From the graph (lower panels) one can see a definite dominance of the chiraleven GPDs. We deduce that $A_{L U}$ is not favored for the extraction of chiral-odd GPDs. The $A_{U T}$ in Fig. 1(b) is quite sensitive to the transversity, however.

\section{Conclusions \& Outlook}

The transversity parton distributions in the nucleon can be accessed through deeply virtual exclusive $\pi^{0}$ meson production. We addressed the feasibility of experimental extraction. This represents a 
consistent quantitative step with respect to our previous work [12]. In particular, only $H_{T}$ and the combination $2 \widetilde{H}_{T}+E_{T}$, were considered, but now they are separated. A similar simplified approach was taken also in Ref.[24] - we differ in the importance attached to the skewedness dependence of $E_{T}, \tilde{E}_{T}$.

We see the results of our extended approach for the many measured and measurable observables. What is especially gratifying is that certain asymmetries constrain the GPDs well enough to separately determine $H_{T}$, and consequently transversity through the limit $H_{T}(x, 0,0)$, and the combination $2 \widetilde{H}_{T}+$ $(1 \pm \xi) E_{T}$.

\section{Acknowledgements}

We are grateful to the organizers of POETIC 6 for a productive meeting in an excellent setting. Work of S.L. supported by U.S. D.O.E. grant DE-FG02-01ER4120.

\section{References}

[1] A. Bacchetta, A. Courtoy and M. Radici, JHEP 1303, 119 (2013).

[2] M. Anselmino, et al. Phys. Rev. D 87, 094019 (2013).

[3] A. Airapetian et al. [ HERMES Collaboration ], Phys. Rev. Lett. 94, 012002 (2005).

[4] COMPASS collaboration, E. Ageev et al., Nucl. Phys. B765, 31 (2007).

[5] Belle collaboration, K. Abe et al., Phys. Rev. Lett. 96, 232002 (2006).

[6] G. R. Goldstein, J. .O. Gonzalez Hernandez and S. Liuti, arXiv:1401.0438 [hep-ph].

[7] R. De Masi, et al., Phys. Rev. C77, 042201 (2008)

[8] G. R. Goldstein, J. .O. Gonzalez Hernandez and S. Liuti, Phys. Rev. D 91, 114013 (2015).

[9] X. D. Ji, Phys. Rev. D 55, 7114 (1997)

[10] P. Hoodbhoy and X. Ji, Phys. Rev. D 58, 054006 (1998).

[11] M. Diehl, Eur. Phys. Jour. C 19, 485 (2001); ibid, Phys. Rept. 388, 41 (2003).

[12] S. Ahmad, G. R. Goldstein and S. Liuti, Phys. Rev. D79, 054014 (2009).

[13] G. R. Goldstein, J. O. Hernandez and S. Liuti, Phys. Rev. D 84, 034007 (2011).

[14] J. O. Gonzalez-Hernandez, S. Liuti, G. R. Goldstein and K. Kathuria, Phys. Rev. C 88, 065206 (2013).

[15] G. R. Goldstein and J. F. Owens, Phys. Rev. D 7 (1973) 865.

[16] G. R. Goldstein, J. O. Hernandez and S. Liuti, J. Phys. G: Nucl. Part. Phys. 39 (2012) 115001.

[17] M. Vanderhaeghen, P. A. M. Guichon and M. Guidal, Phys. Rev. D 60, 094017 (1999).

[18] A. Airapetian et al. [ HERMES Collaboration ], JHEP 0806, 066 (2008); ibid, JHEP 0911, 083 (2009).

[19] F. X. Girod et al. [ CLAS Collaboration ], Phys. Rev. Lett. 100, 162002 (2008).

[20] E. Seder, et al. [CLAS], Phys. Rev. Lett. 114, 032001 (2015); S. Pisano, et al. [CLAS], Phys. Rev. D91, 052014 (2015).

[21] G. D. Cates, C. W. de Jager, S. Riordan and B. Wojtsekhowski, Phys. Rev. Lett. 106, 252003 (2011).

[22] I. Bedlinskiy, et al., Phys.Rev.Lett. 109, 112001(2012).

[23] F. Sabatie, "DVCS \& $\pi^{0}$ electroproduction at Hall A, Jefferson Lab", presentation at CIPANP2015, Vail, Colorado (May 2015).

[24] S. V. Goloskokov, P. Kroll, Eur. Phys. J. A47, 112 (2011). 